Original paper

\title{
Dosimetric comparison of 3-dimensional conformal and field-in-field radiotherapy techniques for the adjuvant treatment of early stage endometrial cancer
}

\author{
Guler Yavas $^{\mathrm{a}, *}$, Cagdas Yavas ${ }^{\mathrm{b}}$, Hilal Acar ${ }^{\mathrm{a}}$, Ahmet Buyukyoruk ${ }^{\mathrm{a}}$, Gokcen Cobanoglu ${ }^{\mathrm{a}}$, \\ Ozlem Secilmis Kerimoglu ${ }^{c}$, Ozlem Yavas ${ }^{\mathrm{d}}$, Cetin Celik ${ }^{\mathrm{c}}$ \\ ${ }^{a}$ Selcuk University, Faculty of Medicine, Department of Radiation Oncology, 42075 Konya, Turkey \\ ${ }^{\mathrm{b}}$ Konya Training and Research Hospital, Department of Radiation Oncology, Turkey \\ ${ }^{c}$ Selcuk University, Faculty of Medicine, Department of Obstetrics and Gynecology, Turkey \\ ${ }^{\mathrm{d}}$ Selcuk University, Faculty of Medicine, Department of Medical Oncology, Turkey
}

\section{A R T I C L E I N F O}

\section{Article history:}

Received 24 July 2012

Received in revised form

30 October 2012

Accepted 12 November 2012

Available online 5 December 2012

\section{Keywords:}

Endometrial cancer

Conformal radiotherapy

Dose-volume histograms

Field-in-field radiotherapy

\begin{abstract}
A B S T R A C T
Background: The purpose of this study is to compare field-in-field radiotherapy (FIF) with conformal radiotherapy (CRT) in terms of dosimetric benefits for early stage endometrial cancer patients. Materials and methods: Ten consecutive early stage endometrial cancer patients who underwent adjuvant external beam radiotherapy were included in the study. For each patient, two different treatment plans were created. FIF and CRT plans were compared for doses in the planning target volume (PTV), the organ at risk (OAR) volumes including rectum, bladder, bowel, bilateral femurs and bone marrow, the dose homogeneity index, and the monitor unit counts required for the treatment.

Results: The FIF technique significantly reduced the maximum dose of the PTV, rectum, bladder, bowel, left femur, right femur and bone marrow ( $p$ values were: $<0.001,0.031,0.003,<0.001,0.001,0.001$ and $<0.001$ respectively). When the OAR volumes irradiated with $>30$ and $>45$ Gy were compared, the results were in favor of the FIF technique. The volumes of rectum, bladder, bowel, left femur, right femur and bone marrow receiving more than the prescription dose of $45 \mathrm{~Gy}$ were significantly reduced with FIF technique ( $p$ values were $0.016,0.039,0.01,0.04,0.037$ and 0.01 respectively). The dose homogeneity index (DHI) was significantly improved with FIF technique $(p<0.001)$.

Conclusions: FIF allowed more homogeneous dose distribution in the PTV and reduced the doses received by OAR. Considering the lower maximum doses in the OAR and PTV, FIF technique seems to be more advantageous than CRT during adjuvant radiotherapy for early stage endometrial cancer patients.
\end{abstract}

(c) 2012 Associazione Italiana di Fisica Medica. Published by Elsevier Ltd. All rights reserved.

\section{Introduction}

Endometrial cancer patients usually present with early symptoms which account for the overall good prognosis associated with the disease. Adjuvant radiotherapy has been shown to improve loco-regional control rates in endometrial cancer patients [1,2]. Adjuvant pelvic radiotherapy is usually recommended for patients with both intermediate-risk and high-risk patients.

Two large randomized controlled trials compared external beam radiotherapy following surgery to no adjuvant therapy in stage I endometrial cancer. GOG-99 phase III trial has included intermediate-risk (International Federation of Gynecology and

\footnotetext{
* Corresponding author. Tel.: +90 332 2244085; fax: +90 3322416065 .

E-mail address: guler.aydinyavas@gmail.com (G. Yavas).
}

Obstetrics (FIGO) 1988 surgical stage IB, IC or occult stage II) endometrial adenocarcinoma patients [2]. The 2-year pelvic recurrence rate was $3 \%$ in the radiation arm versus $12 \%$ in the control arm ( $p$ : 0.007). In the subgroup analysis the difference was particularly evident in patients with (1) poorly differentiated tumor, (2) presence of lymphovascular space invasion and (3) outer third myometrial invasion; age $\geq 50$ years with any two risk factors; or age of at least 70 with any risk factor. The PORTEC-1 (Postoperative Radiation Therapy in Endometrial Carcinoma) study enrolled 714 patients with endometrial cancer who were at intermediate-risk of recurrence or progression (grade 1 with $>50 \%$ myometrial invasion, all grade 2 and grade 3 with $<50 \%$ myometrial invasion) [1]. At 5 years local recurrence was significantly lower for patients who received 46 Gy external beam radiotherapy when compared to the observation arm (4\% versus $14 \%$ ). 
It is generally considered that the clinical target volume (CTV) to be irradiated post-operatively for endometrial cancer includes the draining lymphatics, parametrium and upper vagina [3]. The middle of the pelvis, in the postoperative situation, is often occupied by relatively radio-sensitive small intestine. In addition, rectum and bone marrow are not felt to be at risk for recurrence and hence unnecessary to irradiate.

Radiation therapy causes apoptosis of bone marrow (BM) stem cells and stromal damage, resulting in myelosupression and characteristic pathologic and radiographic BM changes [4]. Clinical studies have shown that radiation induced BM injury depends on both the dose and volume of the BM irradiated [5,6]. Conventional pelvic radiotherapy fields encompass large volumes of hematopoietically active BM, particularly in the pelvis and lower spine. Field-in-field (FIF) radiotherapy technique allows us to decrease excessive doses in both planning target volume (PTV) and organ at risk (OAR) with the same radiation fields. To achieve this dose distribution sub-fields are used. Therefore in the current study we hypothesized that as well as other pelvic organs including rectum, bowel, bone marrow and head of the bilateral femurs, pelvic BM may be spared using FIF technique.

In order to improve dosimetric benefits and spare OAR, several investigators have described the different techniques including intensity modulated RT (IMRT), three non-coplanar conformal fields, field-in-field (FIF) technique [7]. In our clinic whole pelvis RT after surgery has been performed with 3-dimensional conformal radiotherapy. In case of unwanted hot spots which can not be modified with field weighting we use a field-in-field technique. The aim of the FIF technique is to increase dose homogeneity to the planning target volume (PTV) while decreasing the absorbed dose in irradiated tissues outside the targeted tissue. Therefore in the current study we aimed to evaluate the benefit of FIF technique over 3-dimensional conformal radiotherapy technique in early stage endometrial cancer patients.

\section{Material and methods}

\section{Patients}

Ten consecutive patients with histologically confirmed endometrial cancer on FIGO stage IB and II (four of the patients had stage IB disease and remaining six had stage II disease) were enrolled to the study. All the patients underwent total abdominal hysterectomy, bilateral salpingo-oophorectomy and pelvic-paraaortic lymphadenectomy. The median age of the patients was 61.5 years (ranging from 56 to 65 years). Patients were scanned in the supine position and computed tomography (CT) data was acquired with adjacent axial slice spacing of $5 \mathrm{~mm}$, covering the entire pelvis. The data obtained from CT was transferred to the treatment planning system (TPS) (Eclipse, version 8.6; Varian Medical Systems Inc, Palo Alto USA).

\section{Target volumes and organs at risk}

According to the recommendations of consensus guidelines for the delineation of the clinical target volume (CTV) in the postoperative pelvic radiotherapy of endometrial and cervical cancer target volumes were delineated by the same radiation oncologist with the help of an experienced radiologist [3]. CTV included all regions of potential microscopic disease: the surgical bed, regional lymph node areas (common, internal and external iliac nodal groups) as well as the upper $3 \mathrm{~cm}$ of vagina and para-vaginal soft tissue lateral to the vagina. For the location of the nodal groups, a $7 \mathrm{~mm}$ margin around named vessels was used. In case of cervical stromal invasion presacral lymph node-region was also included into the CTV. The CTV was modified to exclude the vertebral body, psoas muscle, rectum, bladder and bowel. A PTV was generated by expanding the CTV $10 \mathrm{~mm}$ isotropically. The whole pelvic field was defined superiorly above the bifurcation of the common iliac vessels and inferiorly $1 \mathrm{~cm}$ above the inferior extent of the obturator foramen. The lateral boarders included a $1.5-2 \mathrm{~cm}$ margin on the pelvic rim $[8,9]$.

Organs at risk (OAR) outlined were bladder, rectum, entire bowel and the bilateral femurs. All of the structures were delineated using pelvic normal tissue contouring guidelines for radiation therapy atlas [10]. The rectum was delineated from the anal margin to the sigmoid flexure. The bladder was contoured from its base to the dome. Peritoneal space occupied or potentially occupied by small and large bowel was contoured as the entire bowel from L4-5 interspace to its lowest extent in the pelvis. The ball of the femur, trochanters and proximal shaft to the level of the bottom of ischial tuberosities was delineated. Total bone marrow (BM) was contoured from the L4 vertebral body to the ischial tuberosities, including the pelvis, L4-5, and sacrum $[5,6]$.

\section{Treatment planning}

All the treatment plans were created by the same medical physicist. Conventional treatment planning was performed using four-field box technique using 18-MV photons designed to treat the whole pelvis. Irregular beam portals of the $4 \mathrm{~F}$ plans were shaped with a multileaf collimator and manually optimized using the beam's eye view technique. The initial calculation of FIF plan was performed with the same fields used for CRT. Subfields blocking hot spot volumes were determined to improve dose homogeneity. The hot spots were determined by careful examination of each axial image by the radiation oncologist. The $105 \%$ and $110 \%$ regions were used to define appropriate blocking of high dose regions. First, for each field a corresponding MLC subfield was created in the beam's eye view projection. After the calculation of fields and subfields the weight of the subfields relative to the open fields were increased until the high dose cloud disappeared. If the resulting maximum dose was still too high, additional subfields and weights were created by the same procedure. Repeating these steps, two to three subfields were created for each conformal field. Normally, some 4.9-6.3 \% of the monitor units of the open fields were delivered in the subfields.

\section{Dosimetric evaluation}

In this study, Varian millennium 80-leaf collimators (MLC) (Varian Medical Systems Inc, Palo Alto USA) were used for both conventional and FIF treatments. The prescribed dose, which was defined as the mean dose in the PTV, was $45 \mathrm{~Gy}$ in 25 fractions at $1.8 \mathrm{~Gy}$ per day. The dose-volume histograms (DVH) were obtained from both plans.

Dose homogeneity in the PTV for CRT and FIF plans was compared by means of the dose homogeneity index (DHI [11],):

$$
\mathrm{DHI}=\frac{\mathrm{D} 2-\mathrm{D} 98}{\mathrm{D} \text { prescription }} \times 100 \%
$$

In this formula, D98 is the maximum dose absorbed in those $2 \%$ of the PTV least irradiated, D2 is the minimum dose absorbed in those $2 \%$ of the PTV most irradiated.

The maximum, minimum and the mean doses of the PTV, rectum, bladder, entire bowel and bilateral femurs and Monitor Unit (MU) settings required for each plan were compared. Tissue volumes in all the critical organs receiving more than 30 and $45 \mathrm{~Gy}$ were also evaluated. 


\section{Statistical analysis}

The Statistical Package for Social Sciences (SPSS) v. 11.0 was used for statistical analysis (SPSS Inc. Chicago, II., USA). Paired samples ttest was used for comparisons. The maximum, minimum and mean doses of PTV and OAR (including bladder, rectum, bowel, BM and head of the bilateral femurs) and the mean volume of PTV and OAR receiving doses in excess of $30 \mathrm{~Gy}$ and $45 \mathrm{~Gy}$ were compared. A $p$ value of $<0.05$ was considered to be significant.

\section{Results}

The mean volumes and standard deviations (SD) of the PTV and OAR are listed in Table 1. The FIF technique allowed more homogeneous dose distributions compared to the CRT technique. The DHI values were $0.0762 \pm 0.011$ and $0.0534 \pm 0.008$ for CRT and FIF techniques respectively ( $p<0.001$ ) (Fig. 1 ).

The maximum dose to the PTV was significantly reduced with the FIF technique $(p<0.001)$ (Fig. 2). However, there wasn't any significant difference in terms of the minimum and mean doses of the PTV (Table 2).

When we compared the maximum, mean and minimum doses of OAR, all of them were in favor of FIF technique. The maximum doses of the OAR including rectum, bladder, bowel, left femur, right femur and BM were significantly decreased with FIF technique. The mean bladder, rectum and bowel doses were significantly reduced with FIF technique. The minimum doses of bladder, bowel and BM were significantly reduced with FIF technique (Table 2).

When the OAR volumes irradiated with $>30$ and $>45 \mathrm{~Gy}$ were evaluated, the results were again in favor of the FIF technique (Table 3). The volumes of bowel and BM receiving $>30 \mathrm{~Gy}$ and the volumes of rectum, bladder, bowel, left femur, right femur and BM receiving $>45$ Gy were significantly decreased with FIF technique.

The mean MU counts \pm SD required for CRT and FIF techniques were $201.2 \pm 6.0$ and $203.2 \pm 5$, respectively. The difference in the average MU values used in the FIF and CRT techniques was not statistically significant $(p=0.61)$.

\section{Discussion}

Risk-adapted adjuvant radiotherapy has been shown to improve local control in patients with early stage endometrial cancer. However, normal tissue toxicities including rectum, bowel, bladder and bone marrow are the main handicap during radiation therapy planning. Several investigators have reported higher genitourinary and gastrointestinal toxicity rates in the irradiated patients [1217]. Thus a radiation oncologist should consider the risk-benefit ratio in recommending adjuvant radiotherapy for early stage endometrial cancer patients.

Table 1

The volumes of the PTV and OAR.

\begin{tabular}{lc}
\hline Volume & Mean volume $\pm \mathrm{SD}^{\mathrm{b}}(\mathrm{cc})$ \\
\hline PTV $^{\mathrm{a}}$ & $1222.1 \pm 279.5$ \\
Rectum & $137.2 \pm 77.7$ \\
Bladder & $188.4 \pm 126.9$ \\
Bowel & $1247.6 \pm 94.7$ \\
Left Femur & $94.1 \pm 12.6$ \\
Right Femur & $95.2 \pm 76.4$ \\
BM $^{\mathrm{c}}$ & $593.2 \pm 162.7$ \\
\hline
\end{tabular}

OAR: Organ at risk.

a PTV: Planning target volume.

b SD: Standard deviation.

c BM: Bone marrow.
In parallel to the developments in the field of radiation oncology the survival times of the patients with gynecologic tumors have been increased as well. It is important to note that there are many more long-term survivors with mild changes in bowel and bladder function that impair quality of life but whose severity do not meet criteria for gradable toxicity. Therefore the term "quality of life" has been increasingly important. In our previous study we have demonstrated that radiation treatment symptoms are the main factors that decrease the quality of life of the patients [18]. Modern radiotherapy techniques allowed us to decrease treatment related toxicities. In our clinic whole pelvis RT after surgery has been performed with 3-dimensional conformal radiotherapy. Therefore we are able to have an idea of the doses received by the entire OAR. However especially in over weight patients unwanted hot spots and excess doses in the OAR are unavoidable. On the other hand endometrial cancer patients are usually overweight. We do not have IMRT software in our clinic. Therefore we evaluated the dosimetric benefits of FIF technique compared with 3D-conformal radiotherapy technique in patients with early stage endometrial cancer. Our results suggested that the FIF technique was superior to 3D-conformal radiotherapy during pelvic radiotherapy in terms of dose homogeneity and the doses received by OAR. FIF technique allowed us to decrease unwanted hot spots.

IMRT has been advocated to improve the therapeutic ratio by conforming more precisely to desired target volumes and achieving steep dose gradients that potentially spare critically important normal structures while producing adequate target coverage. Heron and colleagues compared the conventional 3D-conformal radiotherapy (3D-CRT) in the adjuvant treatment of gynecologic cancer patients [7]. They showed that IMRT reduced the treatment volume for bladder, rectum and small bowel. The mean volume of all bowel, bladder and rectum receiving doses in excess of $30 \mathrm{~Gy}$ was reduced by $36 \%, 66 \%$ and $52 \%$, respectively. In our study although we did not found any significant difference in terms of the mean volume of bladder and rectum receiving doses in excess of $30 \mathrm{~Gy}$, the mean volume of small bowel receiving doses in excess of $30 \mathrm{~Gy}$ was reduced significantly. Moreover the mean volume of bladder, bowel, rectum and bone marrow receiving doses in excess of $45 \mathrm{y}$ was reduced by $10.4 \%, 18 \%, 4.7 \%$ and $19.9 \%$ respectively.

Forrest and colleagues compared the dose to OAR between conventional four-field whole pelvis plan and IMRT plan for cervical cancer patients [19]. Their study demonstrated a statistically significant difference in DHI between the two plans: 1.05 (4F) versus 1.07 (IMRT). Moreover they found significant reduction in the mean V30, V40, V45 and V50 for all OARs (except V30 rectum). The minimum dose to the PTV was 2.45 Gy higher for 4F (45.91 Gy; standard deviation 3.67) compared with IMRT (43.46 Gy; standard deviation 2.65). In our study we also found a statistically significant difference between two plans in terms of DHI. FIF technique allowed us better DHI. When we compared the minimum doses to the PTV, it was higher for FIF technique $(4286.3 \pm 37.1$ for FIF and $4266.8 \pm 16.7$ for 3D-CRT); however the difference wasn't statistically significant ( $p=0.145$ ).

RT may induce local tissue damage that in turn, depending on the severity and the volume affected, may lead to organ dysfunction. Organ dysfunction may be clinical (symptomatic) or subclinical (asymptomatic) [21]. Various Vx values (percentage organ volume receiving $\geq \mathrm{x}$ Gy) are associated with radiation induced normal tissue damage risk. Within individual datasets there are usually strong correlations between the different dosimetric parameters (e.g., V5 and V20), and thus this may partly obscure any "optimal" threshold. Furthermore, the correlations between dosimetric parameters are technique dependent, and readers should carefully assess the similarity of their treatment technique to the historical reports before using any of these limits as clinical 

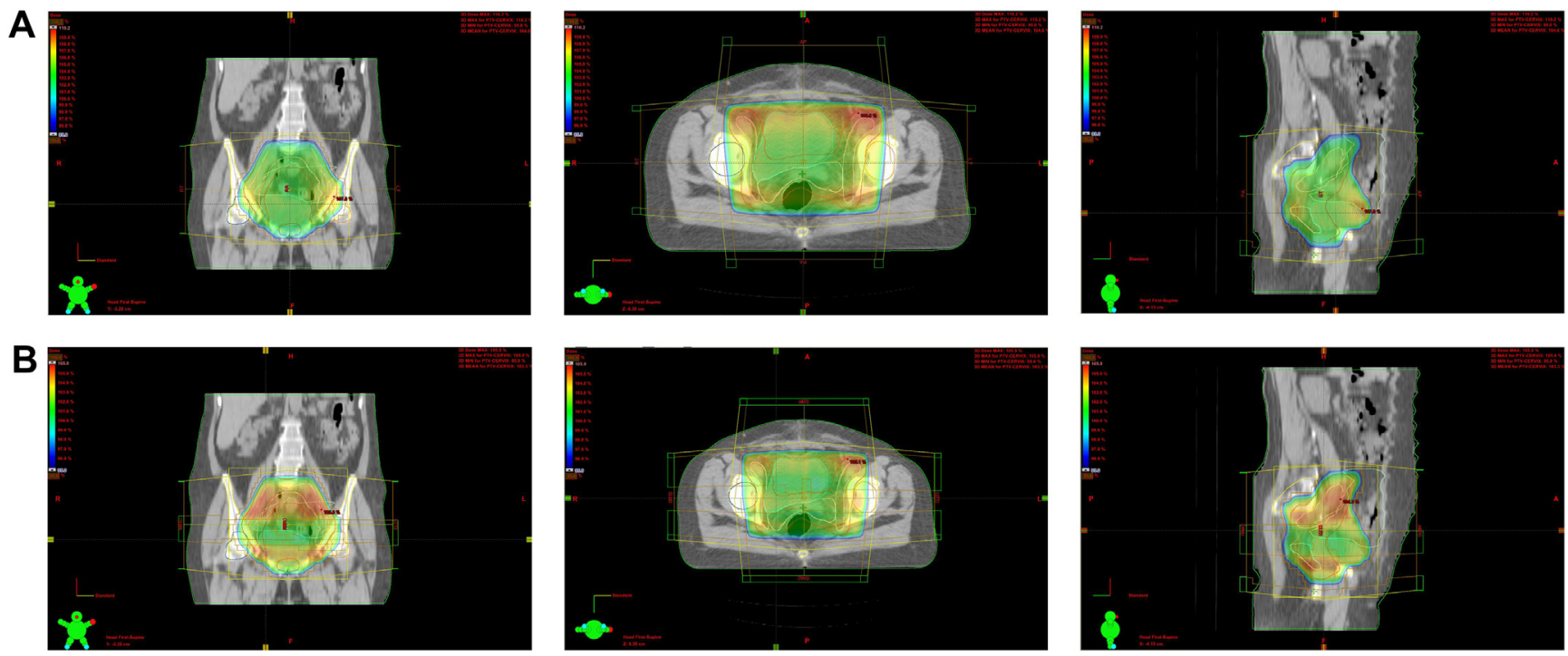

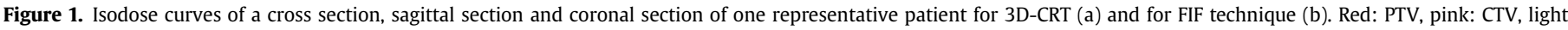

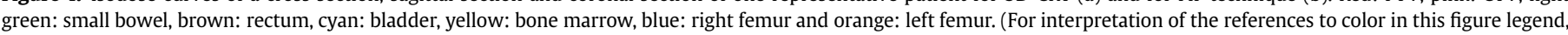
the reader is referred to the web version of this article.)

constraints [20]. Like the similar studies in the literature, in the current study we used V30 and V45 values in order to better define the dose constraints for each OAR $[8,19]$.

Although there are many studies demonstrating the clinical advantage of IMRT in gynecologic patients [5,7,19,22-28], some concerns have been raised about the widespread application of
IMRT $[3,29]$. Due to the presence of steep dose gradients and longer treatment times with IMRT, concerns remain about possible inferior tumor control. The absence of prospective comparisons of IMRT to conventional techniques also makes the clinical significance and cost-efficacy of IMRT uncertain. Finally, an approximate doubling of the risk of second malignancies with IMRT compared to

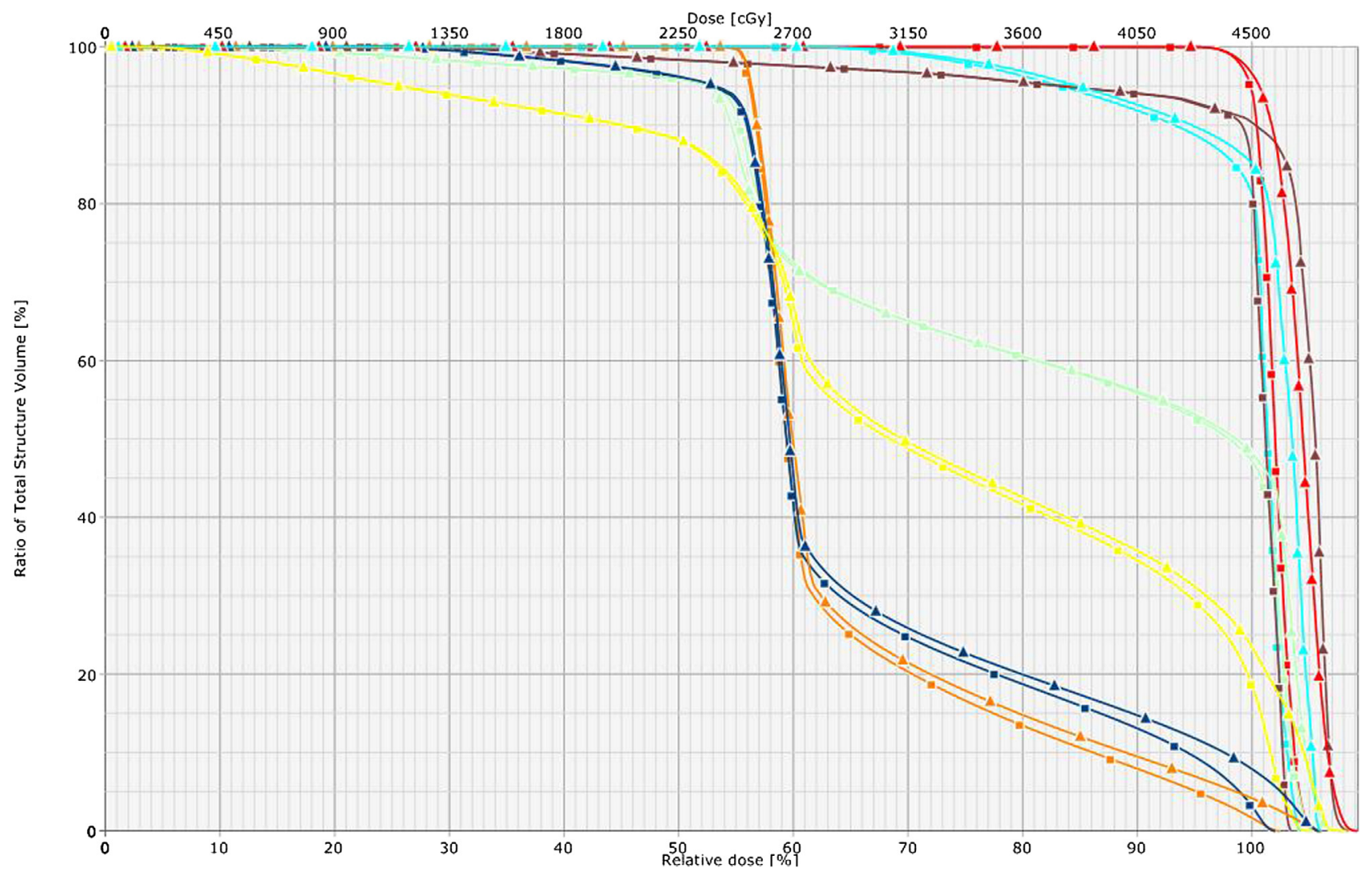

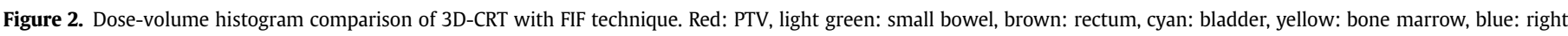
femur and orange: left femur ( $\boldsymbol{\Delta}$ : 3D-CRT, $\mathbf{\square}$ : FIF). (For interpretation of the references to color in this figure legend, the reader is referred to the web version of this article.) 
Table 2

The mean, median and maximum doses of PTV and OAR.

\begin{tabular}{|c|c|c|c|}
\hline Volume & $\mathrm{CRT}^{\mathrm{a}}$ & $\mathrm{FIF}^{\mathrm{c}}$ & $p$ \\
\hline \multicolumn{4}{|c|}{ Maximum Dose $\pm \mathrm{SD}^{\mathrm{d}}(\mathrm{cGy})$} \\
\hline $\mathrm{PTV}^{\mathrm{b}}$ & $4913.4 \pm 46.8$ & $4775.5 \pm 46.7$ & $<0.001^{*}$ \\
\hline Rectum & $4749.2 \pm 73.7$ & $4691.2 \pm 62.6$ & $0.031^{*}$ \\
\hline Bladder & $4797.8 \pm 113.6$ & $4683.4 \pm 63.1$ & 0.003* \\
\hline Bowel & $4852.4 \pm 65.5$ & $4738.7 \pm 83.6$ & $<0.001^{*}$ \\
\hline Left Femur & $4755.8 \pm 76.7$ & $4646.5 \pm 55.4$ & $\mathbf{0 . 0 0 1}^{*}$ \\
\hline Right Femur & $4749.5 \pm 61.7$ & $4628.6 \pm 71.2$ & $0.001 *$ \\
\hline $\mathrm{BM}^{\mathrm{e}}$ & $4845.4 \pm 66.1$ & $4734 \pm 50.3$ & $<0.001^{*}$ \\
\hline \multicolumn{4}{|c|}{ Mean Dose $\pm \mathrm{SD}^{\mathrm{d}}(\mathrm{cGy})$} \\
\hline PTV & $4658.8 \pm 47.8$ & $4623.7 \pm 58.9$ & 0.139 \\
\hline Rectum & $4569.9 \pm 161.6$ & $4462.2 \pm 127.0$ & $0.014^{*}$ \\
\hline Bladder & $4458.0 \pm 293.4$ & $4372.0 \pm 259.8$ & $0.006^{*}$ \\
\hline Bowel & $3148.4 \pm 582.7$ & $3107.6 \pm 117.9$ & $0.006^{*}$ \\
\hline Left Femur & $3024.3 \pm 99.3$ & $2771.0 \pm 40.7$ & 0.112 \\
\hline Right Femur & $3016.2 \pm 121.5$ & $2979.2 \pm 119.4$ & 0.096 \\
\hline $\mathrm{BM}^{\mathrm{e}}$ & $3480.1 \pm 204.6$ & $3374.6 \pm 235.1$ & 0.098 \\
\hline \multicolumn{4}{|c|}{ Minimum Dose $\pm \mathrm{SD}^{\mathrm{d}}(\mathrm{cGy})$} \\
\hline PTV & $4266.8 \pm 16.7$ & $4286.3 \pm 37.1$ & 0.145 \\
\hline Rectum & $2417.1 \pm 188.3$ & $2723.6 \pm 123.7$ & 0.343 \\
\hline Bladder & $2831.1 \pm 627.3$ & $2786.6 \pm 599.1$ & $0.029^{*}$ \\
\hline Bowel & $181.0 \pm 91.5$ & $177.8 \pm 79.1$ & $0.006^{*}$ \\
\hline Left Femur & $1638.2 \pm 276.9$ & $1171.0 \pm 590.1$ & 0.176 \\
\hline Right Femur & $1334.1 \pm 121.5$ & $1219.2 \pm 175.8$ & 0.405 \\
\hline $\mathrm{BM}^{\mathrm{e}}$ & $183.2 \pm 69.6$ & $181.1 \pm 69$ & 0.019* \\
\hline
\end{tabular}

Bold values signify $p<0.05$

*Statistically significant.

a CRT: Conformal Radiotherapy.

b PTV: Planning target volume.

c FIF: Field-in-field.

d SD: Standard Deviation.

e BM: Bone Marrow.

conventional techniques has been hypothesized [3,30], due to increased total body dose from leakage radiation and the increased volume of tissue exposed to low dose radiation. Also there are limited studies reporting long-term outcomes.

There are some shortcomings of the current study. First, there is a relative lack of data on organ motion, particularly as it relates to pelvic and abdominal structures. Organ motion and patient setup uncertainty are important considerations with conformal planning in gynecologic patients. However, the targets for adjuvant radiotherapy for gynecologic malignancies are less likely to be mobile. Because the vagina is not attached to the bladder as in the normal state, the movement as a result of bladder filling is likely significantly diminished [8]. Although no consensus planning margins for

Table 3

The mean V30 and V45 values of OAR (cc).

\begin{tabular}{|c|c|c|c|}
\hline Volume & $\mathrm{CRT}^{\mathrm{a}} \%$ & FIF $^{\mathrm{b}} \%$ & $p$ \\
\hline \multicolumn{4}{|l|}{ V30 } \\
\hline Rectum & 97.7 & 97.4 & 0.496 \\
\hline Bladder & 94.2 & 94 & 0.509 \\
\hline Bowel & 47.4 & 46 & $0.003^{*}$ \\
\hline Left Femur & 34.4 & 32.0 & 0.211 \\
\hline Right Femur & 30.50 & 28.10 & 0.083 \\
\hline $\mathrm{BM}^{\mathrm{c}}$ & 59.4 & 58.7 & $0.01^{*}$ \\
\hline \multicolumn{4}{|l|}{ V45 } \\
\hline Rectum & 82.4 & 78.5 & $0.016^{*}$ \\
\hline Bladder & 80.4 & 72.0 & 0.039* \\
\hline Bowel & 27.2 & 22.3 & $0.01^{*}$ \\
\hline Left Femur & 10.10 & 8.10 & $0.04^{*}$ \\
\hline Right Femur & 10.10 & 6.0 & $0.037^{*}$ \\
\hline $\mathrm{BM}^{\mathrm{c}}$ & 29.7 & 23.8 & $0.01^{*}$ \\
\hline
\end{tabular}

Bold values signify $p<0.05$

*Statistically significant.

a CRT: Conformal Radiotherapy.

b FIF: Field-in-field.

c BM: Bone Marrow.
3-D and IMRT treatment have been created, a $1.0-1.5 \mathrm{~cm}$ (or institution-specific) uniform CTV expansion is commonly advocated. We also created the PTVs by expanding the CTV $10 \mathrm{~mm}$ isotropically. According to the report 50 of the International Commission on Radiation Units and Measurements (ICRU-50) [31], PTV is defined as the volume usually created by extending CTV $0.5-$ $1 \mathrm{~cm}$, accounting for factors such as internal organ motion, set-up variation and patient movement. Therefore, we have already given the suggested margin in order to compensate for the internal organ motion. Moreover, we established a treatment protocol to standardize the treatment for these patients. All the patients were treated with full-bladder and empty rectum. During daily treatment we evaluated digitally reconstructed radiographs (DRR) for each patient by observing bony anatomy provided from the radiotherapy.

In our study, all of the structures were delineated using pelvic normal tissue contouring guidelines for radiation therapy atlas [10]. We intentionally did not outline specific loops of bowel, but instead we contoured the peritoneal space occupied or potentially occupied by small and large bowel from L4-5 interspace to its lowest extent in the pelvis. This methodology, as others have found, is more likely to overestimate the dose to small bowel as represented in the DVH since the probability of small bowel residing in a specific region of the pelvis is variable from day to day $[8,21]$.

In our study, we did not consider the body mass index (BMI) of our patients. However, we intentionally chose overweight patients, because during pelvic radiotherapy planning we encountered difficulties particularly for these patients. The depth to the tumor is larger compared to normal-weight patients, which can result in unwanted hot spots and excess doses to OARs.

In conclusion, in the adjuvant radiation treatment of early stage endometrial cancer patients, FIF technique enables significant increase in dose homogeneity in the PTV while allowing significant reduction in the doses received by OAR. Considering the dosimetric advantage of the FIF technique, this technique may be an alternative treatment option during pelvic radiotherapy in clinics which do not have IMRT.

\section{Conflict of interest}

There is no conflict of interest.

\section{Funding}

None.

\section{References}

[1] Creutzberg CL, van Putten WL, Koper PC, Lybeert ML, Jobsen JJ, WárlámRodenhuis CC, et al. Surgery and postoperative radiotherapy versus surgery alone for patients with stage-I endometrial carcinoma: multicentre randomized trial. PORTEC Study Group. Post operative radiation therapy in endometrial carcinoma. Lancet 2000;55:1404-11.

[2] Keys HM, Roberts JA, Brunetto VL, Zaino RJ, Spirtos NM, Bloss JD, et al. A phase III trial of surgery with or without adjunctive external pelvic radiation therapy in intermediate risk endometrial adenocarcinoma; a gynecologic oncology group study. Gynecol Oncol 2004;92:744-51.

[3] Small Jr W, Mell LK, Anderson P, Creutzberg C, De Los Santos J, Gaffney D, et al. Consensus guidelines for delineation of clinical target volume for intensitymodulated pelvic radiotherapy in postoperative treatment of endometrial and cervical cancer. Int J Radiat Oncol Biol Phys 2008 Jun 1;71(2):428-34.

[4] Mauch P, Constine L, Greenberger J, Knospe W, Sullivan J, Liesveld JL, et al. Hematopoietic stem cell compartment: acute and late effects of radiation therapy and chemotherapy. Int J Radiat Oncol Biol Phys 1995;31:1319-39.

[5] Mell LK, Schomas DA, Salama JK, Devisetty K, Aydogan B, Miller RC, et al. Association between bone marrow dosimetric parameters and acute hematologic toxicity in anal cancer patients treated with concurrent chemotherapy and intensity modulated radiotherapy. Int J Radiat Oncol Biol Phys 2008;70: $1431-7$ 
[6] Rose BS, Aydogan B, Liang Y, Yeginer M, Hasselle MD, Dandekar V, et al. Normal tissue complication probability modeling of acute hematologic toxicity in cervical cancer patients treated with chemo radiotherapy. Int J Radiat Oncol Biol Phys 2011;79:800-7.

[7] Heron DE, Gerszten K, Selvaraj RN, King GC, Sonnik D, Gallion H, et al. Convetional 3D conformal versus intensity-modulated radiotherapy for the adjuvant treatment of gynecologic malignancies: a comparative dosimetric study of dose-volume histograms. Gynecol Oncol 2003;91:39-45.

[8] Martinez-Monge R, Fernandes PS, Gupta N, Gahbauer R. Cross-sectional nodal atlas: a tool for the definition of clinical target volumes in three-dimensional radiation therapy planning. Radiology 1999;211:815-28.

[9] Mak AC, Rich TA, Schultheiss TE, Kavanagh B, Ota DM, Romsdahl MM. Late complications of postoperative radiation therapy for cancer of the rectum and rectosigmoid. Int J Radiat Oncol Biol Phys 1994;28:597-603.

[10] Gay HA, Barthold HJ, O’Meara E, Bosch WR, El Naqa I, Al-Lozi R, et al. Pelvic normal tissue contouring guidelines for radiation therapy: a radiation therapy oncology group consensus panel atlas. Int J Radiat Oncol Biol Phys 2012 Jul 1;83(3):e353-62.

[11] Wu Q, Mohan R, Morris M, Lauve A, Schmidt-Ullrich R. Simultaneous integrated boost intensity modulated radiotherapy for locally advanced head and neck squamous cell carcinomas. Dosimetric results. Int J Radiat Oncol Biol Phys 2003;56:573-85.

[12] Roeske JC, Mundt AJ, Halpern H, Sweeney P, Sutton H, Powers C, et al. Late rectal sequelae following definitive radiation therapy for carcinoma of the uterine cervix: a dosimetric analysis. Int J Radiat Oncol Biol Phys 1997;37: $351-8$.

[13] Snijders-Keilholz A, Griffioen G, Davelaar J, Trimbos JB, Leer JW. Vitamin B12 malabsorption after irradiation for gynecological tumors. Anticancer Res 1993; $13: 1877-81$

[14] Einhorn N. Frequency of severe complications after radiation therapy for cervical carcinoma. Acta Radiol 1974;14:42-8.

[15] Aalders J, Abeler V, Kolstad P, Onsrud M. Postoperative external irradiation and prognostic parameters in stage I endometrial carcinoma: clinical and histopathological study of 540 patients. Obstet Gynecol 1980;56:419-27.

[16] Kinsella TJ, Bloomer WD. Tolerance of the intestine to radiation therapy. Surg Gynaecol Obstet 1980;151:273-84.

[17] Meerwaldt JH, Hoekstra CJ, van Putten WL, Tjokrowardojo AJ, Koper PC. Endometrial adenocarcinoma, adjuvant radiotherapy tailored to prognostic factors. Int J Radiat Oncol Biol Phys 1990;18:299-304.

[18] Yavas G, Dogan NU, Yavas C, Benzer N, Yuce D, Celik C. Prospective assessment of quality of life and psychological distress in patients with gynecologic malignancy: a 1-year prospective study. Int J Gynecol Cancer 2012 Jul;22(6): 1096-101.
[19] Forrest J, Presutti J, Davidson M, Hamilton P, Kiss A, Thomas GA. Dosimetric planning study comparing intensity-modulated radiotherapy with four-field conformal pelvic radiotherapy for the definitive treatment of cervical carcinoma. Clin Oncol 2012;24:63-70.

[20] Marks LB, Yorke ED, Jackson A, Ten Haken RK, Constine LS, Eisbruch A, et al Use of normal tissue complication probability models in the clinic. Int J Radiat Oncol Biol Phys 2010;76(3) [supplement 10-19].

[21] Nuyttens JJ, Robertson JM, Yan D, Martinez A. The influence of small bowel motion on both a conventional three-field and intensity modulated radiation therapy (IMRT) for rectal cancer. Cancer Radiother 2004;8(5):297-304.

[22] Ahamad A, D'Souza W, Salehpour M, Iyer R, Tucker SL, Jhingran A, et al. Intensity modulated radiation therapy after hysterectomy: comparison with conventional treatment and sensitivity of the normal-tissue-sparing effect to margin size. Int J Radiat Oncol Biol Phys 2005;62:1117-24.

[23] Chan P, Yeo I, Perkins G, Fyles A, Milosevic M. Dosimetric comparison of intensity modulated, conformal, and four-field pelvic radiotherapy boost plans for gynecologic cancer: a retrospective planning study. Radiat Oncol 2006;4: 13-21.

[24] Du XL, Tao J, Sheng XG, Lu CH, Yu H, Wang C, et al. Intensity-modulated radiation therapy for advanced cervical cancer: a comparison of dosimetric and clinical outcomes with conventional radiotherapy. Gynecol Oncol 2012 Apr;125(1):151-7.

[25] Mundt AJ, Mell LK, Roeske JC. Preliminary analysis of chronic gastrointestinal toxicity in gynecology patients treated with intensity-modulated whole pelvic radiation therapy. Int J Radiat Oncol Biol Phys 2003;56:1354-60.

[26] Portelance L, Chao KS, Grigsby PW, Bennet H, Low D. Intensity-modulated radiation therapy (IMRT) reduces small bowel, rectum, and bladder doses in patients with cervical cancer receiving pelvic and para-aortic irradiation. Int J Radiat Oncol Biol Phys 2001;51:261-6.

[27] Salama JK, Mundt AJ, Roeske J, Mehta N. Preliminary outcome and toxicity report of extended-field, intensity-modulated radiation therapy for gynecologic malignancies. Int J Radiat Oncol Biol Phys 2006;65:1170-6.

[28] Brixey CJ, Roeske JC, Lujan AE, Yamada SD, Rotmensch J, Mundt AJ. Impact of intensity-modulated radiotherapy on acute hematologic toxicity in women with gynecologic malignancies. Int J Radiat Oncol Biol Phys 2002;54:1388-96.

[29] Randall ME, Ibbott GS. Intensity-modulated radiation therapy for gynecologic cancers: pitfalls, hazards, and cautions to be considered. Semin Radiat Oncol 2006; $16: 138-43$.

[30] Hall EJ. Intensity-modulated radiation therapy, protons, and the risk of second cancers. Int J Radiat Oncol Biol Phys 2006;65:1-7. [PubMed: 16618572].

[31] International Commission on Radiation Units and Measurements (ICRU). Prescribing, recording and reporting photon beam therapy. Report 50 Washington, DC: ICRU; 1993. 